\title{
Characterisation of Performances of Thermoelectric GENERATORS FOR ENERGY HARVESTING APPLICATIONS
}

\author{
Petar Gljušćić, ${ }^{1,2}$ Saša Zelenika ${ }^{1,2}$ * \& Ervin Kamenar ${ }^{1,2}$ \\ ${ }^{1}$ University of Rijeka, Faculty of Engineering, Vukovarska 58, 51000 Rijeka, Croatia \\ ${ }^{2}$ University of Rijeka, Centre for Micro- and Nanosciences and Technologies, \\ Radmile Matejčić 2, 51000 Rijeka, Croatia \\ * Corresponding author: sasa.zelenika@ riteh.hr
}
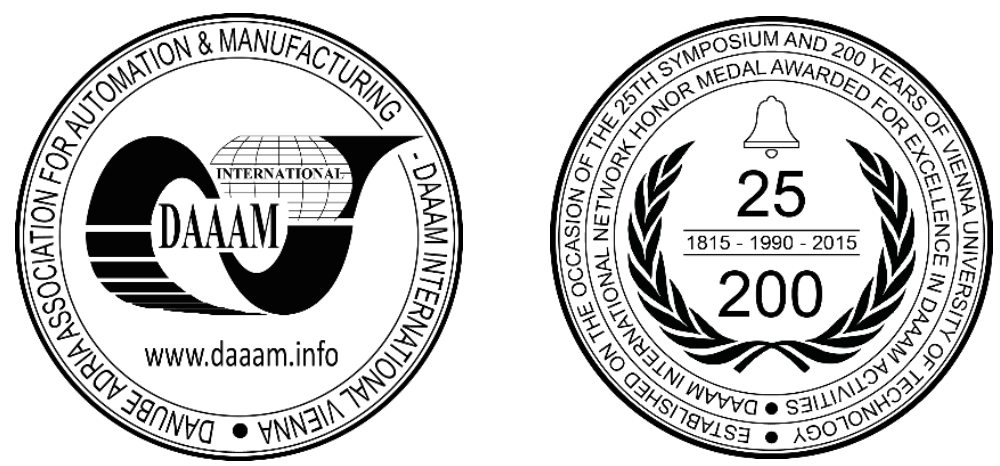

This Publication has to be referred as: Gljuscic, P[etar]; Zelenika, S[asa] \& Kamenar, E[rvin] (2018). Characterisation of Performances of Thermoelectric Generators for Energy Harvesting Applications, Proceedings of the 29th DAAAM International Symposium, pp.0025-0030, B. Katalinic (Ed.), Published by DAAAM International, ISBN 978-3-90273420-4, ISSN 1726-9679, Vienna, Austria

DOI: $10.2507 / 29$ th.daaam.proceedings.004

\begin{abstract}
One of the most common ambient energy sources suitable for energy harvesting is waste heat emitted by machines, from hot pipes, from human and animal bodies (i.e., resulting from metabolism) or from radioactive materials. Heat emanated from humans is particularly interesting for energy harvesting applications, since it is possible to use it as a power source for wearable devices - and in particular for medical sensors. On the other hand, thermoelectric generators (TEGs) allow converting waste heat into electrical energy via the Seebeck effect. An experimental setup is developed in this work with the aim of charactering the performances of three types of commercially available TEG devices. Heat is hence brought into the system on the hot side of the TEGs, while it is dissipated via a heatsink on their cold side. Temperatures on both sides are measured with thermocouples and by using thermal imaging, while, concurrently, various electrical loads are connected to the harvesters. Multimeters are employed to measure the resulting electrical parameters (voltage and current), enabling the determination of the power output and the efficiency of the studied devices. The acquired data provide thus means of matching the analysed TEGs to the respective working conditions in adequate applications.
\end{abstract}

Keywords: energy harvesting; thermoelectric generator; Seebeck effect; efficiency; experimental characterization

\section{Introduction}

As one of the consequences of their rapid development, the size of widely used electronic devices is shrinking to the level that they can be worn on the body. Such devices, also known as wearables, usually comprise various types of sensors (e.g. medical diagnostic devices, fall detectors or drug delivery systems) with the corresponding data elaboration and communication devices. 
Wearable devices require a wearable power source as well. Besides the conventional batteries, which in several applications can hinder the autonomy of the wearable devices, the needed power can often be generated on the body itself, i.e., it can be based on energy harvesting principles. In fact, energy harvesting is the process of collecting low-level ambient energy and converting it into electrical energy to be used for powering miniaturized autonomous devices, sensor networks, wearable electronics or Internet-of-Things components. Devices powered via energy harvesting, being batteryless, are smaller and therefore more convenient for application in areas where miniaturisation is a prerequisite. An environmental energy source that can be efficiently used to power wearable technology devices, and is commonly used in energy harvesting technology, is waste heat [1], [2], [3], [4]. In effect, thermal energy, i.e., waste heat, is emanated by multiple sources such as machines (due to combustion and friction), pipes transferring hot medium, radioactive materials, but also from human and animal bodies due to metabolism. The latter heat source is particularly interesting for applications in wearable technologies. Commonly, thermoelectric generators (TEGs) are then used to convert waste heat into electrical energy. As shown on Figure 1, TEGs take advantage of the Seebeck effect to generate electrical voltage from the available heat flow. A TEG generally comprises a large number of mutually connected $p$ - and $n$ - type semiconductor pellets, acting as thermocouples. The value that largely influences the efficiency of a TEG is the temperature difference $\Delta T$ between the hot and the cold side of the device. On the other hand, the material itself, typically for lower temperature differences generally bismuth-telluride (BiTe) or lead-telluride (PbTe) are used, needs to have thermoelectric properties and a high thermoelectric figure of merit $z T$ that correlates directly with the efficiency of the device [1], [2].

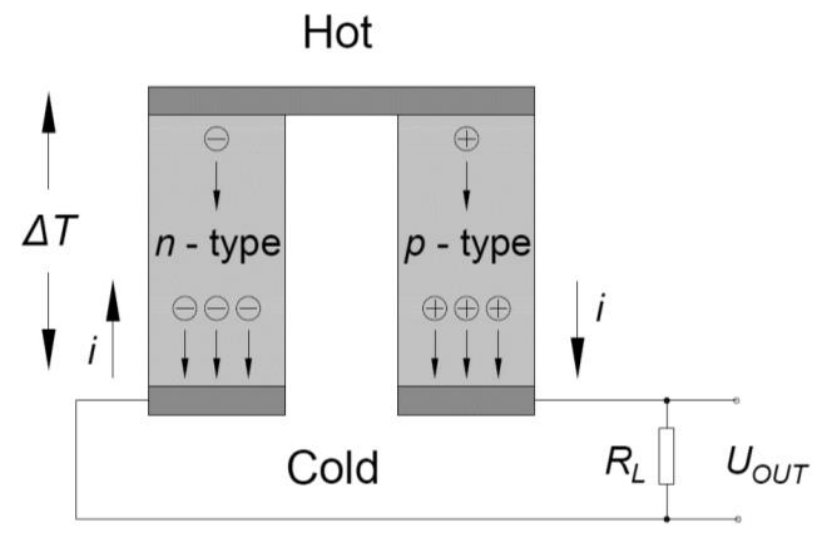

Fig. 1. Seebeck effect in a thermocouple of a thermoelectric generator

In the herein considered context of the potential application of TEGs for wearable devices, the main drawback of using TEGs to collect waste body heat is the low available temperature difference $\Delta T$ between the skin and the environment. This generally means that in such conditions a typical commercial TEG will work below its optimal efficiency. On the other hand, most of the data provided by the manufacturers of TEG devices is focused on achieving high efficiencies at higher $\Delta T$ values. The development of new thermoelectric materials (usually nanostructured exotic alloys) also strives towards the same objective.

The goal of this work is hence to perform the experimental assessment of the performances of three commercially available TEGs at lower temperatures, thus validating their suitability to be applied for energy harvesting of waste heat from the human body, i.e., as an energy source for wearable devices aimed at powering medical sensors.

\section{Experimental set-up}

Three types of TEGs, provided by the same TECTEG MRF ${ }^{\circledR}$ manufacturer [6], each optimized for particular operating conditions and applications - meaning that each model has a maximum power output at a specific combination of temperature, load resistance and voltage values, are analysed in this work. The TEG models used in the experiments are:

- TEG2-126LDT - 40 × 40 × $5.45 \mathrm{~mm}^{3}$ BiTe TEG optimized for lower temperature differences [7];

- TEG2-07025HT-SS - 40 × 40 × $5.45 \mathrm{~mm}^{3}$ BiTe TEG optimized for liquid-to-liquid applications [8];

- TEG1-PB-12611-6.0 - 56 x 56 x $5.45 \mathrm{~mm}^{3}$ BiTe and PbTe hybrid, capable of withstanding higher temperature differences (up to $360{ }^{\circ} \mathrm{C}$ ) [9].

To characterise the performances of a TEG device (position 3 in Figures 2 and 3 depicting the used experimental setup), a controlled simulation of working conditions is needed. This is achieved in this work by applying heat to the hot side of the device, constituted by an aluminium block (position 1), employed to distribute uniformly the heat to the surface of the TEG device, by using a $320 \mathrm{~W}$ and 24 1/min digital SMD hot air soldering station ZD939L [10] - see Figure 4. On the other hand, heat is dissipated from the cold side via a heat sink (position 5) complemented with an axial fan (position 6). The temperatures are measured on both sides of the TEG using Fluke 116 digital multimeters [11] equipped, given the range of values of the temperature differences typical for the considered energy harvesting application, with Fluke 80BKA K-type thermocouples having an accuracy of $+/-2,2^{\circ} \mathrm{C}$ [12] (positions 2 and 4 in Figures 2 and 3 ). 
The output voltage of the device is measured via a custom-developed LabVIEW ${ }^{\circledR}$ virtual instrument (VI) [13] coupled to a suitable National Instruments PXI-6221 data acquisition card (DAQ) [14], where the attained electric current is calculated over a minute $0.87 \Omega$ resistor $\left(R_{\mathrm{P}}\right)$ by applying Ohm's law. Due to the low voltage values measured on the $R_{\mathrm{P}}$ resistor, the signal is amplified by a factor 10 by using an amplifier. To enable the calculation of output powers and the respective energy conversion efficiencies, the TEG is connected also to a variable resistor $R_{\mathrm{L}}$ with resistance values up to $100 \Omega$, which simulates the effective load of the foreseen wearable medical sensors.

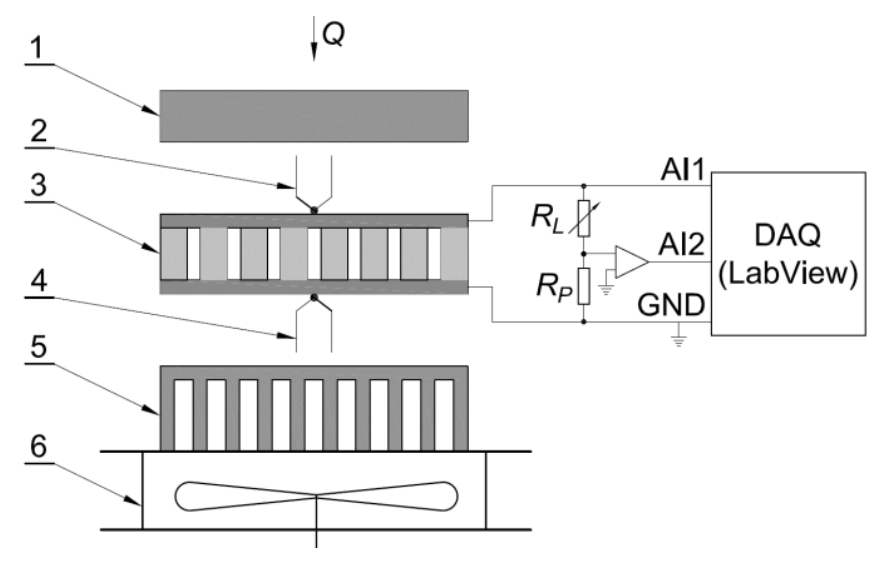

Fig. 2. Scheme of the experimental set-up

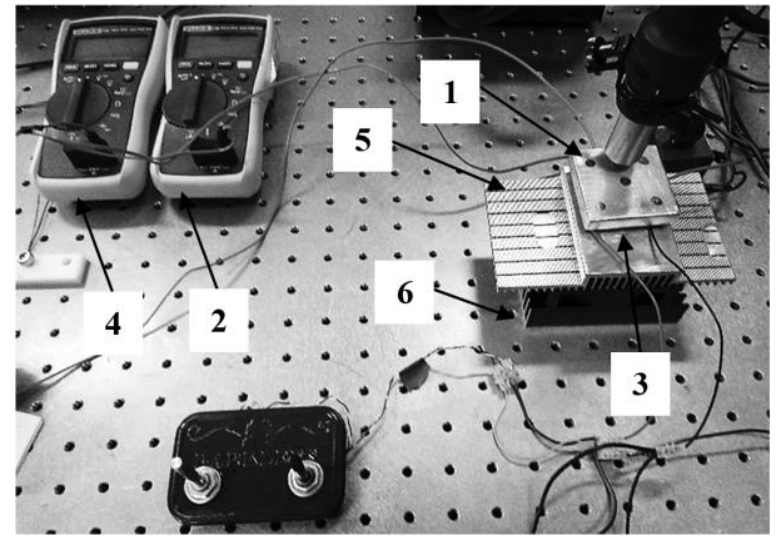

Fig. 3. Actual appearance of the experimental set-up

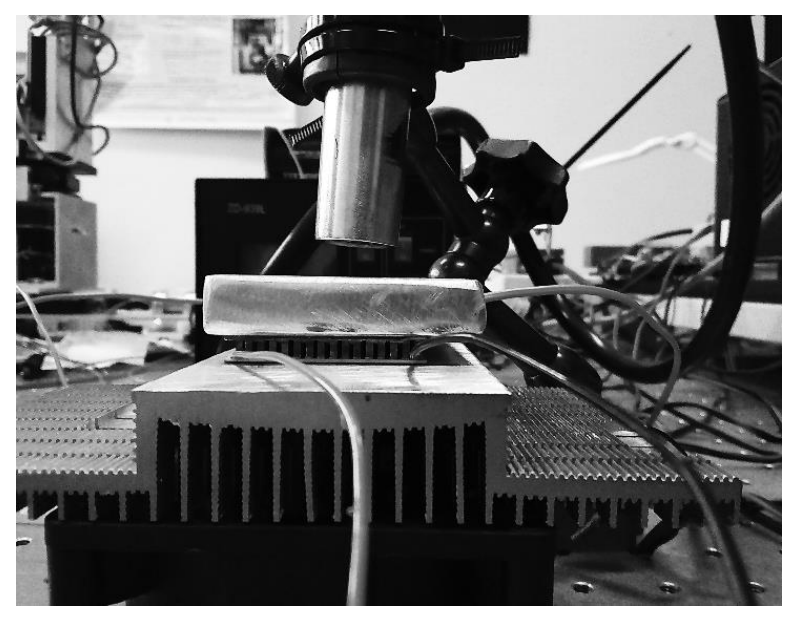

Fig. 4. Magnified detail of the TEG

The output electrical parameters are hence measured vs. the hot-to-cold side temperature differences ranging from $20{ }^{\circ} \mathrm{C}$ up to $80{ }^{\circ} \mathrm{C}$, with $10^{\circ} \mathrm{C}$ increments, while the temperature of the cold side is kept at a constant value of $\sim 30{ }^{\circ} \mathrm{C}$. To get a better monitoring of the temperature distribution across the elements of the set-up, the experiments are recorded also by using a FLIR ThermaCAM P65 HS thermal imaging camera [15]. The resulting temperature distribution from the heated aluminium block, across the TEG and down to the cooled heat sink can thus be clearly seen in Figure 5.

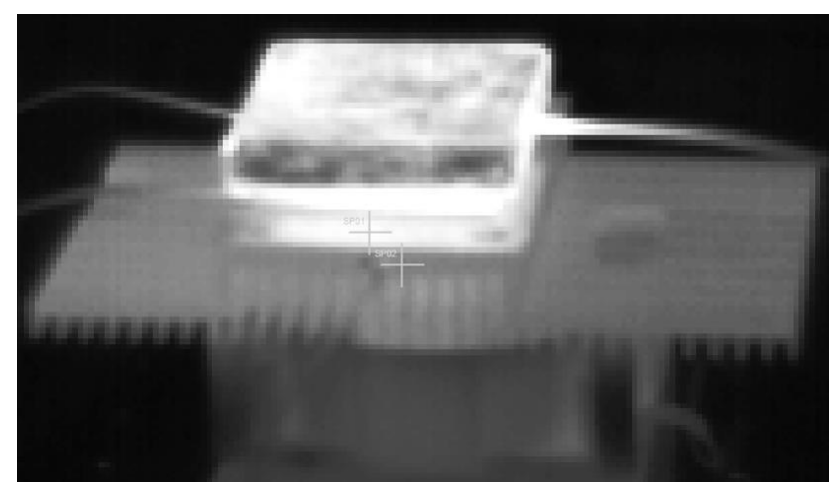

Fig. 5. Tracking of the thermal distribution in the experimental set-up via the infrared camera 


\section{Measurements results}

For a given induced temperature difference $\Delta T$, a voltage is generated on the used TEG device and the resulting current in calculated. For a determined $\Delta T$, the thus attained output powers $P_{\mathrm{EL}}=U^{2} / R_{\mathrm{L}}$ on the device vs. the applied load resistances $R_{\mathrm{L}}$ are hence shown in the graphs depicted in Figures 6,8 and 10 for the respectively used TEGs. It can be noticed that, as expected, the obtained maximal powers correspond to the $R_{\mathrm{L}}$ values that match the internal resistance of the TEG itself [16]. The output power is then relatively high in a narrow area around this peak, decreasing rapidly for both higher and lower $R_{\mathrm{L}}$ values - although a more rapid decrease can be noticed for $R_{\mathrm{L}}$ values lower than the internal resistance. On the other hand, the respective $P_{\mathrm{EL}}$ vs. current $I$ dependencies are shown in Figures 7, 9 and 11.

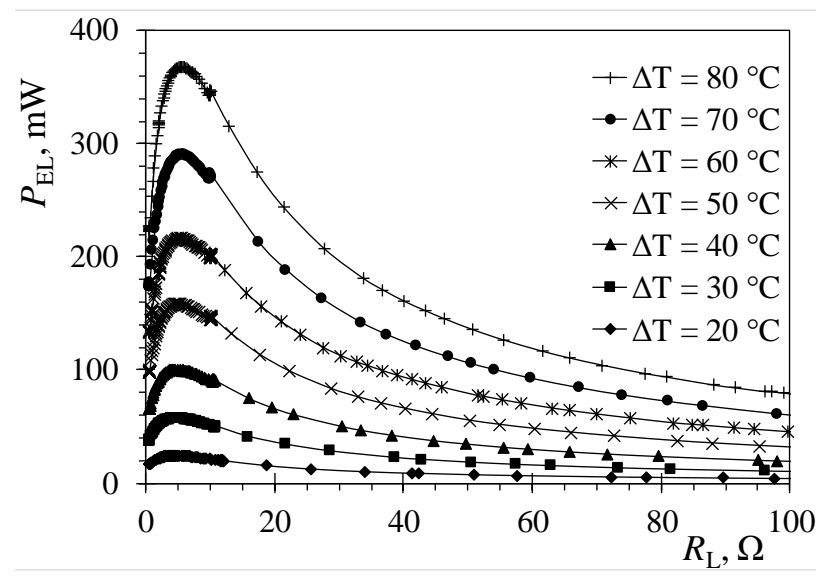

Fig. 6. TEG2-126LDT: $P_{\mathrm{EL}}$ vs. $R_{\mathrm{L}}$ for varying $\Delta T$

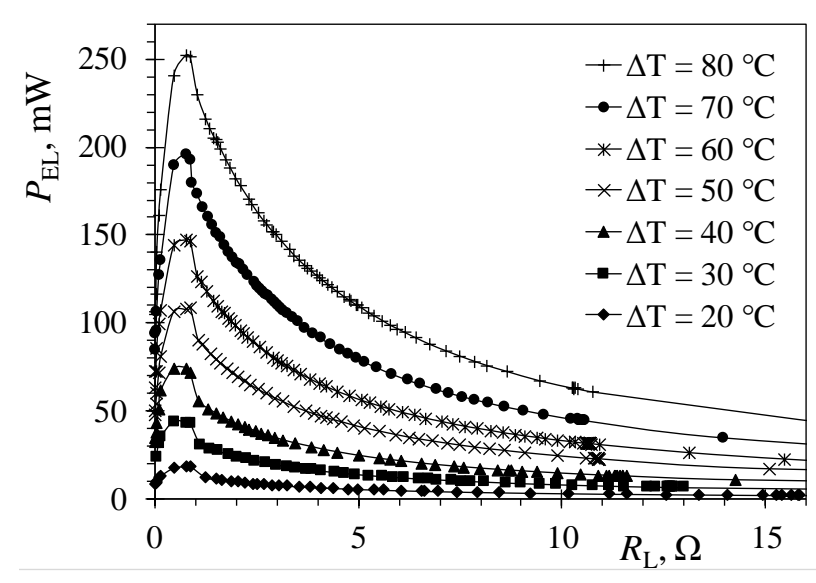

Fig. 8. TEG2-07025HT-SS: $P_{\mathrm{EL}}$ vs. $R_{\mathrm{L}}$ for varying $\Delta T$

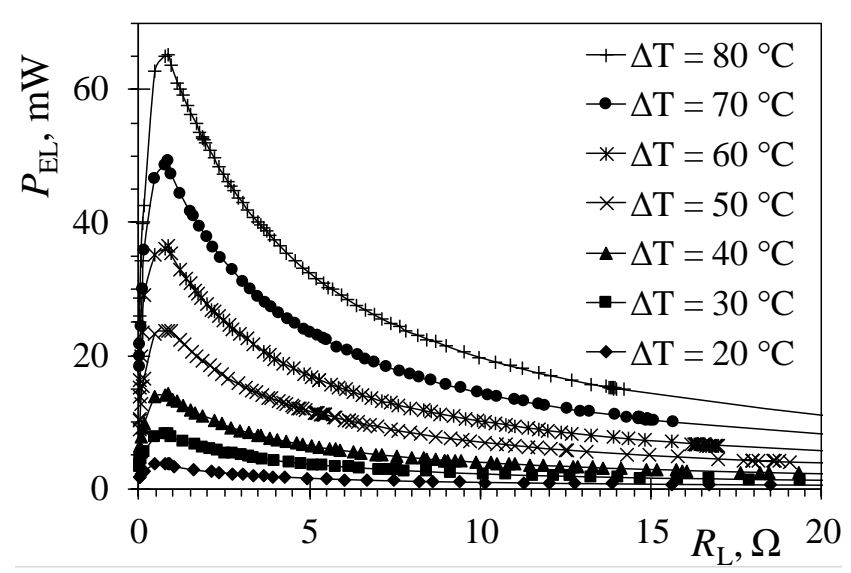

Fig. 10. TEG1-PB-12611-6.0: $P_{\mathrm{EL}}$ vs. $R_{\mathrm{L}}$ for varying $\Delta T$

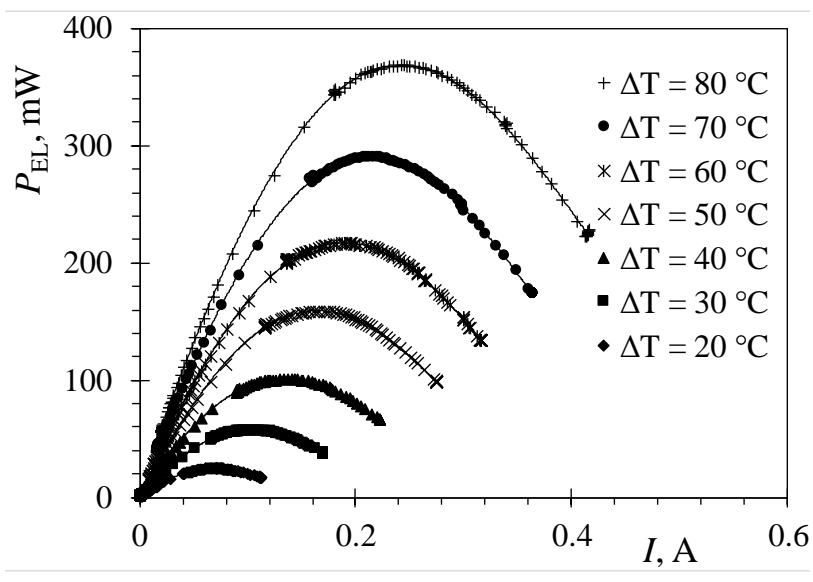

Fig. 7. TEG2-126LDT: $P_{\mathrm{EL}}$ vs. $I$ for varying $\Delta T$

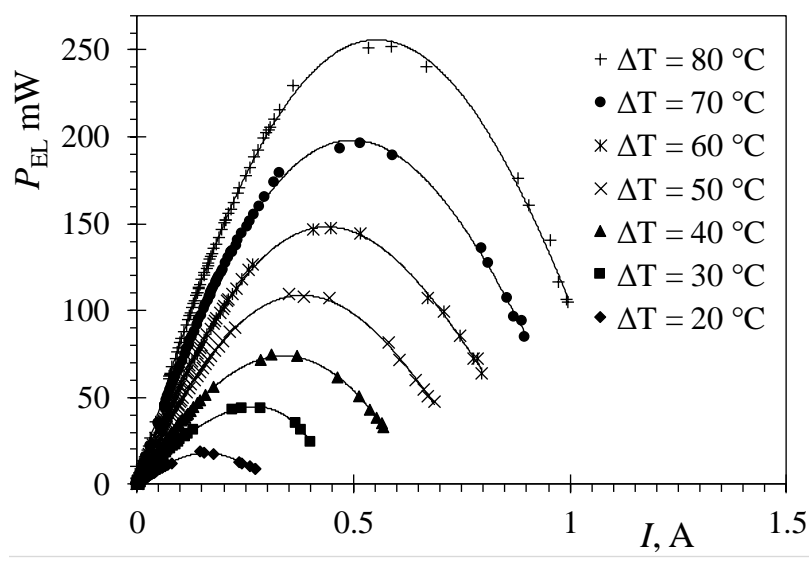

Fig. 9. TEG2-07025HT-SS: $P_{\mathrm{EL}}$ vs. $I$ for varying $\Delta T$

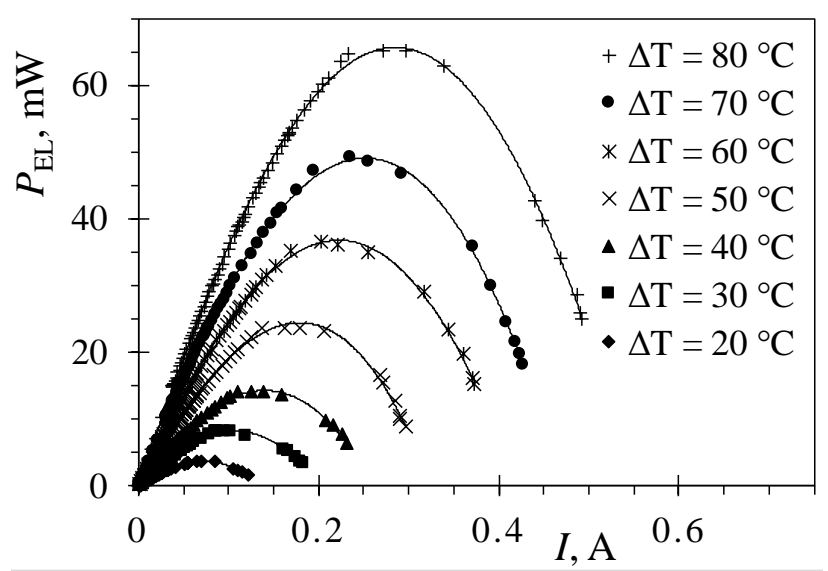

Fig. 11. TEG1-PB-12611-6.0: $P_{\mathrm{EL}}$ vs. $I$ for varying $\Delta T$ 
The obtained $P_{\mathrm{EL}}$ values allow also calculating the efficiencies $\eta_{\mathrm{TEG}}$ of the studied TEG devices, which, as already pointed out, depend on the temperature differences $\Delta T$ and the figure of merit $z T$ of the respective thermoelectric material. In fact, $\eta_{\text {TEG }}$ can be determined by comparing the heat flow $P_{\mathrm{Q}}$ through the TEG device and the respective output electrical power $P_{\mathrm{EL}}$ depicted in the above figures. The heat flow $P_{\mathrm{Q}}$ is herein estimated by measuring the surface temperature on both sides of the aluminium block used as the hot side of the analysed device for each of its nominal temperatures, and calculating the resulting heat transferred via conduction through this block. The surface temperatures are measured in this frame in five different points of the relevant surfaces of the block by using an infrared noncontact thermometer. The thus obtained maximal efficiencies $\eta_{\text {TEG }}$ of the studied TEGs, with the respective maximal output powers $P_{\text {EL }}$, as well as maximal voltages $U$ and currents $I$, as measured at the maximum considered temperature difference $\Delta T$, are shown in Table 1. The maximum output parameters for a temperature difference of $\Delta T=20^{\circ} \mathrm{C}$, which is more relevant to human body waste heat energy harvesting, are in turn shown in Table 2.

\begin{tabular}{|c|c|c|c|c|}
\hline TEG & $\boldsymbol{U}_{\max }, \mathbf{V}$ & $\boldsymbol{I}_{\max }, \mathbf{A}$ & $\boldsymbol{P}_{\text {EL_max }}, \mathbf{m W}$ & $\boldsymbol{\eta}_{\text {TEG_max }}, \boldsymbol{\%}$ \\
\hline TEG2-126LDT & 2.93 & 0.416 & 368.4 & 0.11 \\
\hline TEG2-07025HT-SS & 0.886 & 0.996 & 251.9 & 0.08 \\
\hline TEG1-PB-12611-6.0 & 0.486 & 0.492 & 65.3 & 0.02 \\
\hline
\end{tabular}

Table 1. TEGs' maximal outputs and efficiencies for the maximum considered temperature difference $\Delta T=80{ }^{\circ} \mathrm{C}$

\begin{tabular}{|c|c|c|c|c|}
\hline TEG & $\boldsymbol{U}_{\max }, \mathbf{V}$ & $\boldsymbol{I}_{\max }, \mathbf{A}$ & $\boldsymbol{P}_{\text {EL_max }}, \mathbf{m W}$ & $\boldsymbol{\eta}_{\text {TEG_max }}, \boldsymbol{\%}$ \\
\hline TEG2-126LDT & 0.353 & 0.071 & 24.98 & 0.0076 \\
\hline TEG2-07025HT-SS & 0.13 & 0.144 & 18.72 & 0.0060 \\
\hline TEG1-PB-12611-6.0 & 0.086 & 0.064 & 3.78 & 0.0011 \\
\hline
\end{tabular}

Table 2. TEGs' maximal outputs and efficiencies for $\Delta T=20^{\circ} \mathrm{C}$

It is evident here that the configuration of the analysed TEGs has, as expected, a marked influence on the obtained maximal powers and efficiencies. In fact, the TEG2-126LDT device, whose design has been optimized for lower operating temperatures, allows obtaining the highest maximal output power $P_{\mathrm{EL} \_ \text {max }}=368.4 \mathrm{~mW}$ as well as the highest maximal efficiency $\eta_{\text {TEG_max }}=0.11 \%$. These values are obtained for a resistance value $R_{\mathrm{L}}=5.6 \Omega$ (see Figure 6) and for a current value of $I=0.242$ A (i.e., different from the maximal current value $I_{\max }=0.416 \mathrm{~A}-\mathrm{cf}$. Figure 7 ). As expected, for a lower temperature difference, the respective maximal values $P_{\mathrm{EL} \_ \text {max }}$ and $\eta_{\mathrm{TEG} \_ \text {max }}$ decrease considerably but for the TEG2126LDT device are still markedly higher than those of the other considered TEGs.

When the TEG2-07025HT-SS TEG is considered, the maximal output power value is $P_{\text {EL_max }}=251.9 \mathrm{~mW}$ (at $R_{\mathrm{L}}=$ $0.77 \Omega$ and $I=0.586 \mathrm{~A}$ - see Figures 8 and 9 ), and the respective peak efficiency is $\eta_{\text {TEG } \_ \text {max }}=0.08 \%$. Albeit these peak values are lower than for the TEG2-126LDT device, they are still relatively high, considering that TEG2-07025HT-SS was optimised for higher optimal operating temperatures.

The hybrid BiTe-PbTe TEG1-PB-12611-6.0 device, optimised for even higher operating temperatures, results, for the low temperatures used in the experiments, in the lowest powers and efficiencies.

\section{Conclusions and outlook}

The goal of this work is the experimental characterisation of the performances of commercially available thermoelectric generators as human body energy harvesting power sources for wearable medical devices. A suitable experimental set-up is therefore developed and in-depth measurements are carried out on three TEG models at varying temperature differences as well as load resistance values. This enables to study the behaviour of the considered devices in controlled operating conditions similar to those found in energy harvesting applications.

Based upon the measurement results and the power needs of wearable devices, it is concluded that commercially available TEGs, especially those optimized for low temperature applications - such as the TEG2-126LDT device, enable providing power levels suitable to power typical wearable medical devices. The study needs, however, to be supplemented with the testing of the TEGs also in real operating condition, i.e., when the factual heat from a human body in different states of exertion is used.

In future work, a practical application of thermoelectric generators as energy harvesters in wearable devices will thus be pursued in collaboration with medical institutions. In fact, TEGs coupled to medical sensors and suitable electrical components able to acquire, analyse and transmit the data, could be worn as clothing or as accessories, while being completely autonomous. What is more, wearable devices are especially interesting in the field of telemedicine, where they could be used for real-time remote monitoring of patients, enabling thus early warning in cases of an aggravation of patients' physiological states, such as e.g. blood pressure, pulse or body temperature. Similar systems could also enable the potential use of autonomous devices for immediate drug delivery [3], [4]. Since the typical medical wearable devices' power consumptions ranges are in the domain of tens up to a couple of hundred $\mathrm{mW}$ [5], the results attained in this work substantiate, in fact, the assumption that TEGs could be considered in this frame a viable power source. 
Future work will, in any case, have to rely also on further theoretical analyses, as well as corresponding experimental testing of these or other devices [17] apt to be used as wearable energy sources. It is important to mention here also that a collaboration initiated with the deep X-ray lithography (DXRL) laboratory at the Elettra synchrotron radiation facility in Trieste, Italy, could allow also investigating in this frame the possibility of using the LIGA technology (German acronym for Lithographie, Galvanoformung, Abformung, i.e., lithography, electroplating and moulding [18], [19]) in manufacturing innovative and optimized energy harvesting devices. All of this could eventually allow the creation of integrated autonomous wearable medical devices based on innovative energy harvesting design configurations.

\section{Acknowledgments}

The work described in this paper is enabled by using the equipment funded via the ERDF project RC.2.2.06-0001 "Research Infrastructure for Campus-based Laboratories at the University of Rijeka - RISK" as well as via the support of the University of Rijeka grant 13.09.1.2.09 "Characterisation and Modelling of Materials and Devices for Innovative Applications".

\section{References}

[1] Kaźmierski, T. J. \& Beeby, S. (Ed.) (2011). Energy harvesting systems principles, modeling and applications, Springer, ISBN: 978-1-4419-7565-2, New York, NY, USA

[2] Prya, S. \& Inman, D. (Ed.) (2009). Energy harvesting technologies, Springer, ISBN: 978-0-387-76463-4, New York, NY, USA

[3] Musalek, M. (2017). A wearable fall detector for elderly people, Proceedings of the $28^{\text {th }}$ DAAAM International Symposium, Zadar, Croatia, ISSN: 1726-9679, ISBN: 978-3-902734-11-2, Katalinic, B. (Ed.), pp. 1015-1020, DAAAM International, Vienna, Austria, DOI: 10.2507/28th.daaam.proceedings.141

[4] Prausnitz, M. R. (2004). Microneedles for transdermal drug delivery. Advanced Drug Delivery Reviews, Vol. 56, No. 5, pp. 581-587, ISSN: 0169-409X

[5] Chen, A. (2011). Thermal Energy Harvesting with Thermoelectrics for Self-powered Sensors with Applications to Implantable Medical Devices, Body Sensor Networks and Aging in Place, Ph.D. Dissertation, University of California, Berkeley, CA, USA

[6] https://tecteg.com/ (2016). TECTEG MRF, Accessed 2018-06-20

[7] http://tecteg.com/wp-content/uploads/2015/11/SPEC-SHEET-TEG2-126LDT.pdf (2018). Tecteg TEG2-126LDT for Body \& Sensor Power Thermoelectric Harvesting Applications, Accessed 2018-01-20

[8] http://tecteg.com/wp-content/uploads/2014/09/SpecTEG2-07025HT-SS.pdf (2018). Tecteg Specifications TEG Module TEG2-07025HT-SS, Accessed 2018-01-20

[9] http://tecteg.com/wp-content/uploads/2015/01/TEG1-PB-12611-6.0_CBH-1-Final-November-17th-update.pdf (2018). Tecteg MODULE TEG1-PB-12611-6.0 - Operating parameters, Accessed 2018-01-20

[10] https://www.chipoteka.hr/artik1/96330/lemna-stanica-za-smd-zd-939-1-vruci-zrak-150-5000c-7300083502 (2018). Chipoteka, Accessed on 2018-06-18

[11] http://en-us.fluke.com/products/digital-multimeters/fluke-116-digital-multimeter.html (2018). Fluke 116 Digital Multimeter, Accessed on 2018-05-25

[12] http://en-us.fluke.com/products/all-accessories/fluke-80bk-a.html (2018). Fluke 80BK-A Integrated DMM Temperature Probe, Accessed on 2018-05-25

[13] http://www.ni.com/en-rs/shop/labview/labview-details.html (2018). LabVIEW, Accessed on 2018-06-11

[14] http://www.ni.com/en-rs/support/model.pxi-6221.html (2018). National Instruments PXI-6221 Multifunction I/O Module, Accessed on 2018-05-18

[15] http://www.testequipmentdepot.com/flir/pdf/p65hs_manu.pdf (2006). FLIR Systems ThermaCAM P65 HS - User's manual, Accessed 2018-05-16

[16] Cartwright, K. V. (2008). Non-calculus derivation of the maximum power transfer theorem. Technology Interface, Vol. 8, No. 2, pp. 1-19, ISSN: 1523-9926

[17] Gljuscic, P. \& Zelenika, S. (2018). Coupled electromechanical numerical modelling of piezoelectric vibration energy harvesters, these proceedings

[18] Madou, M. J. (2002). Fundamentals of Microfabrication - The Science of Miniaturisation $-2^{\text {nd }}$ ed., CRC Press, ISBN: 9781482274004, Boca Raton, FL, USA

[19] Chen, Q. H.; De Bona, F.; Matteucci, M.; Savoia, A. \& Zelenika, S. (1995). LIGA Beamline at ELETTRA: Conceptual Design and Technical Specifications, Project study performed on behalf of the MicroMore Company, Trieste (I) 